\title{
The osteogenic or adipogenic lineage commitment of human mesenchymal stem cells is determined by protein kinase $\mathrm{C}$ delta
}

\author{
Sooho Lee ${ }^{1}$, Hee-Yeon Cho ${ }^{1,2}$, Hang Thi Thuy Bui ${ }^{1,2}$ and Dongchul Kang ${ }^{1,2^{*}}$
}

\begin{abstract}
Background: Mesenchymal stem cells (MSCs) have the potential to differentiate into specialized cell lineages such as osteoblasts and adipocytes in vitro. There exists a reciprocal relationship between osteogenic and adipogenic differentiation of MSCs that an osteogenic phenotype occurs at the expense of an adipogenic phenotype and vice versa, which in turn influence one another's phenotype through negative feedback loops. Thus, it is important to understand what signaling molecules modulate the lineage commitment of MSCs. Protein kinase C (PKC) plays a central role in cellular signal transduction for mediating diverse biological functions, and dysregulation of PKC activity is involved in various metabolic diseases including cancer, diabetes, and heart disease. Although the role of individual PKC isoforms has been investigated in various fields, the potential role of PKC in bone metabolism is not completely understood. In this study, we investigated the potential role of PKC $\delta$ in osteogenic lineage commitment of human bone marrow-derived mesenchymal stem cells (hBMSCs).

Results: We observed that expression and phosphorylation of PKC $\delta$ were increased during osteogenic differentiation of hBMSCs. Pharmacological inhibition and genetic ablation of PKC in hBMSCs resulted in a significant attenuation of osteogenic differentiation as evidenced by reduced ALP activity and ECM mineralization, as well as down-regulation of the expression of osteoblast-specific genes. These effects were also accompanied by induction of adipogenic differentiation and up-regulation of the expression of adipocyte-specific genes involved in lipid synthesis in osteogenic induction of hBMSCs. Additionally, the activation of AMPK, which is a key cellular energy sensor, induced osteogenesis of hBMSCs. However, the inhibition of AMPK activity by compound C did not affect the activation of PKC $\delta$ at all, indicating that there is no direct correlation between AMPK and PKC $\delta$ in osteogenesis of hBMSCs.
\end{abstract}

Conclusions: These results suggest that PKC $\delta$ is a critical regulator for the balance between osteogenesis and adipogenesis of hBMSCs and thus has a potential novel therapeutic target for the treatment of metabolic bone diseases.

Keywords: hBMSCs, Osteogenic differentiation, Signal transduction, PKC $\delta$, AMPK

\section{Background}

Human mesenchymal stem cells (hMSCs), also known as adult multipotent stem cells, have been identified in the bone marrow and in various tissues such as adipose tissue, synovial tissue, periosteum, perichondrium and cartilage [1]. These cells have the capacity of self-renewal and the potential to differentiate into specialized cell lineages,

\footnotetext{
* Correspondence: dckang@hallym.ac.kr

'Illsong Institute of Life Science, Hallym University, Anyang, Gyeonggi-do 431-060, Republic of Korea

2Department of Biomedical Gerontology, Graduate School of Hallym University, Chuncheon, Gangwon-do 200-702, Republic of Korea
}

including osteoblasts, adipocytes and chondrocytes under permissive conditions [2]. Importantly, the potential of these cells in cell-based regenerative therapies hold tremendous promise for the treatment of various diseases including osteogenesis imperfecta, cardiovascular disease, and neurological disease [3,4]. Hence, it is important to understand the regulatory mechanism responsible for their differentiation.

Mature osteoblasts, which terminally differentiate into osteocytes, play an essential role for the initiation of bone mineralization and formation, leading to increased bone regeneration rate. Mineralized bone matrix is considered 
to be a hallmark of the final phase of osteogenic differentiation [5]. These processes are tightly controlled by the expression of osteogenesis-related genes, including alkaline phosphatase (ALP), which is not restricted to osteogenic cells and is also expressed in other cell types including embryonic stem cells, runt-related transcription factor 2 (RUNX2), osteocalcin (OCN), and osterix (OSX) [6]. Bone formation is dependent on the recruitment of an adequate number of osteoblasts and their osteogenic activity [7]. However, the impaired bone formation, which is functionally associated with decreased osteoblastic bone-forming activity, contributes to the pathogenesis of metabolic bone diseases including osteoporosis, osteomalacia, and Paget's disease [8]. Therefore, understanding the molecular mechanisms underlying bone formation has emerged as a potential therapeutic approach for the treatment of these diseases.

Protein kinase $\mathrm{C}$ (PKC) is a family of serine/threonine protein kinases that is known to be involved in a multitude of physiological processes such as cell proliferation, differentiation, apoptosis, and survival. The PKC family consists of at least 11 distinct isoforms in mammals. PKCs are classified into three groups depending on their structure and cofactor requirements: $\mathrm{Ca}^{2+} /$ diacylglycerol (DAG)-dependent classical PKC ( $\alpha, \beta 1, \beta 2$ and $\gamma)$, DAGdependent novel PKC $(\delta, \varepsilon, \eta, \theta$ and $\mu)$, and $\mathrm{Ca}^{2+} / \mathrm{DAG}$ independent atypical PKC $(\lambda / \mathrm{s}$ and $\zeta)$. The PKC activity is also tightly regulated by its association with protein complexes and its intracellular distribution [9-12]. Recently, several studies have reported that specific PKC isoforms are involved in embryonic bone formation and remodeling by affecting both osteoblast and osteoclast activity [13-16]. These findings indicate that PKC could be targeted to drive osteogenesis in hMSCs. However, the exact mechanism of osteogenic differentiation of hMSCs regulated by PKC is still not fully understood.

In addition, the AMPK signaling pathway, a master regulator of cellular energy homeostasis, is involved in bone metabolism. Activation of AMPK stimulates bone formation in vitro, while the lack of either $\alpha$ or $\beta$ subunit of AMPK results in reduced bone mass in mice [17]. AMPK has been reported as an upstream kinase of PKC $\delta$ in various cell types $[18,19]$. Although these studies suggest the possibility that AMPK/PKC $\delta$ pathway could participate in the MSC differentiation, there are no reports available as yet of the interrelationship between AMPK and $\mathrm{PKC} \delta$ during differentiation into osteogenic lineage.

The aim of our study was to determine the role of specific PKC isoform in osteogenic differentiation. In this study, we employed human bone marrow-derived mesenchymal stem cells (hBMSCs). We identified that unlike other PKC isoforms, $\mathrm{PKC} \delta$ mRNA and protein levels steadily increased during osteogenic differentiation of hBMSCs and further examined the role of PKC $\delta$ in the regulation of their osteogenic differentiation. We found that both pharmacological and genetic inhibition of PKC $\delta$ impaired osteogenic differentiation of hBMSCs, including a decreased ALP activity and matrix mineralization, as well as the down-regulation of osteogenic marker gene expression. Interestingly, we found that activation of AMPK, similar to changes in $\mathrm{PKC} \delta$ expression, induced osteogenesis of hBMSCs. However, there was no direct correlation between PKC $\delta$ and AMPK under our experimental condition. We further showed that the effect of PKC $\delta$ inhibition on hBMSC osteogenic differentiation was exerted through a positive regulation of adipogenic differentiation. Notably, both pharmacological and genetic inhibition of PKC $\delta$ in hBMSCs exhibited more adipogenic phenotype than their counterparts under osteogenic condition. Thus, these findings demonstrate the potential importance of PKC $\delta$ in directing hBMSC differentiation and provide a promising new avenue for the treatment of metabolic bone diseases.

\section{Methods}

\section{Cell culture}

All cell culture media and supplements were obtained from Gibco (Carlsbad, CA, USA), unless otherwise indicated. Human bone marrow-derived mesenchymal stem cells (hBMSCs) were purchased from ScienCell Research Laboratories (Cat. No. 7500; Carlsbad, CA, USA) and maintained in growth medium (GM) consisting of $\alpha$ Minimum Essential Medium ( $\alpha$-MEM) supplemented with $16.5 \%$ fetal bovine serum (FBS) and antibiotics (100 units $/ \mathrm{mL}$ penicillin and $100 \mu \mathrm{g} / \mathrm{mL}$ streptomycin) at $37^{\circ} \mathrm{C}$ in a humidified atmosphere of $5 \% \mathrm{CO}_{2}$ and $95 \%$ air. Cells between passages 3 and 10 were used for all experiments. For lentivirus production, HEK293T cells were cultured in Dulbecco's modified Eagle's medium (DMEM) containing $10 \% \mathrm{FBS}$ and antibiotics (100 units $/ \mathrm{mL}$ penicillin and $100 \mu \mathrm{g} / \mathrm{mL}$ streptomycin) at $37^{\circ} \mathrm{C}$ in a humidified atmosphere of $5 \% \mathrm{CO}_{2}$ and $95 \%$ air.

\section{Osteogenic induction and alizarin red S staining}

For osteogenic differentiation, hBMSCs were plated at density of $3 \times 10^{5}$ cells/well on 6 -well plates or $1 \times 10^{4}$ cells/well on 96-well plates. After 2 days of incubation at which $100 \%$ confluent, hBMSCs were cultured for an additional 14 days in osteogenic differentiation medium (ODM) consisting of $\alpha$-MEM supplemented with $10 \%$ FBS, $100 \mathrm{nM}$ dexamethasone (Sigma-Aldrich, St. Louis, MO, USA), $10 \mathrm{mM} \beta$-glycerophosphate (Sigma-Aldrich, St. Louis, MO, USA), $50 \mu \mathrm{M}$ ascorbic-2-phosphate (SigmaAldrich, St. Louis, MO, USA), 100 units $/ \mathrm{mL}$ penicillin and $100 \mu \mathrm{g} / \mathrm{mL}$ streptomycin and then treated with either vehicle (DMSO; Sigma-Aldrich, St. Louis, MO, USA), or rottlerin (Calbiochem, La Jolla, CA, USA), or compound C (Calbiochem, La Jolla, CA, USA), respectively. Fresh 
medium was changed twice per week. Osteogenic differentiation of hBMSCs was assessed by alizarin red S staining for the presence of calcium deposits. Briefly, the cells were washed twice with PBS (Sigma-Aldrich, St. Louis, MO, USA), fixed with $4 \%$ formaldehyde (Sigma-Aldrich, St. Louis, MO, USA) for $30 \mathrm{~min}$ at room temperature, rinsed with distilled water, and then stained with $2 \%(\mathrm{w} / \mathrm{v})$ alizarin red S (Sigma-Aldrich, St. Louis, MO, USA) dissolved in distilled water $(\mathrm{pH} 4.2$; adjusted with $10 \%$ ammonium hydroxide [Sigma-Aldrich, St. Louis, MO, USA]) for $45 \mathrm{~min}$. Cells were then washed extensively with distilled water and examined for mineralization of extracellular matrix (ECM). After imaging, the dye was eluted with $10 \%(\mathrm{w} / \mathrm{v})$ cetylpyridinium chloride monohydrate (SigmaAldrich, St. Louis, MO, USA) in $10 \mathrm{mM}$ sodium phosphate (pH 7.0; Sigma-Aldrich, St. Louis, MO, USA) for $1 \mathrm{~h}$ at room temperature, and the absorbance was measured at $570 \mathrm{~nm}$ using a Multiskan ${ }^{\mathrm{mi}} \mathrm{GO}$ microplate reader (Thermo Scientific, Waltham, MA, USA).

\section{Oil red $O$ staining}

The hBMSCs were cultured in ODM as described above for 14 days. Accumulation of lipid droplets in differentiated adipocytes from hBMSCs was assessed by oil red $\mathrm{O}$ staining. Briefly, cells were washed twice with PBS and fixed with $4 \%$ formaldehyde for $30 \mathrm{~min}$ at room temperature. After washing two times with PBS, cells were stained with $0.6 \%$ oil red O (Sigma-Aldrich, St. Louis, MO, USA) in isopropanol (Sigma-Aldrich, St. Louis, MO, USA) for $2 \mathrm{~h}$ at room temperature. The stain was then removed, and the cells were rinsed five times with distilled water. The stained lipid droplets were observed with an inverted phase-contrast microscope (Olympus, Tokyo, Japan).

\section{ALP activity assay}

Cellular ALP activity as an early marker of osteogenic differentiation was assessed at day 7. Cells were washed twice with PBS and then lysed with protein lysis buffer containing $50 \mathrm{mM}$ Tris- $\mathrm{HCl} \mathrm{pH}$ 7.4 (Promega, Madison, WI, USA), $150 \mathrm{mM} \mathrm{NaCl}$ (Sigma-Aldrich, St. Louis, MO, USA), 1 mM EDTA (Sigma-Aldrich, St. Louis, MO, USA), and 1\% NP-40 (Sigma-Aldrich, St. Louis, MO, USA). ALP activity was determined colorimetrically by incubating protein lysates with the substrate $p$-nitrophenyl phosphate (Sigma-Aldrich, St. Louis, MO, USA) in a 96-well plate at $37^{\circ} \mathrm{C}$ for $30 \mathrm{~min}$. The absorbance was measured at $405 \mathrm{~nm}$ and normalized against the corresponding protein concentrations. The values were expressed as fold change relative to undifferentiated cells.

\section{RNA extraction and reverse transcription PCR (RT-PCR)}

Total RNA was extracted from cells using Trizol reagent (Invitrogen, Carlsbad, CA, USA), and cDNA was reversetranscribed from $2 \mu \mathrm{g}$ of total RNA with the GoScript ${ }^{\text {tw }}$
Reverse Transcription System (Promega, Madison, WI, USA) according to the manufacturer's instructions. The primer sequences used for PCR are given in Additional file 1: Table S1. The PCR was performed as follows: one cycle of $3 \mathrm{~min}$ at $95^{\circ} \mathrm{C} ; 35$ cycles of denaturation at $95^{\circ} \mathrm{C}$ for $30 \mathrm{sec}$, annealing at $56^{\circ} \mathrm{C}$ for $30 \mathrm{sec}$ and extension at $72^{\circ} \mathrm{C}$ for $45 \mathrm{sec}$; and then a final cycle of $5 \mathrm{~min}$ at $72^{\circ} \mathrm{C}$. The PCR products were loaded onto $1 \%$ agarose gel containing ethidium bromide (Promega, Madison, WI, USA). The expression data were normalized to $\beta$-actin mRNA levels in each sample.

\section{Western blot analysis}

Cells were washed twice with PBS and lysed in RIPA lysis buffer including $50 \mathrm{mM}$ Tris- $\mathrm{HCl}$ pH 7.4, $150 \mathrm{mM} \mathrm{NaCl}$, 1 mM EDTA, 1\% NP-40, 0.1\% SDS (USB, Cleveland, OH, USA), $0.5 \%$ sodium deoxycholate (Sigma-Aldrich, St. Louis, MO, USA), 1 mM PMSF (Sigma-Aldrich, St. Louis, MO, USA), protease inhibitor cocktail (Pierce Biotechnology, Rockford, IL, USA), and phosphatase inhibitors containing $10 \mathrm{mM}$ sodium fluoride (Sigma-Aldrich, St. Louis, MO, USA), $2 \mathrm{mM}$ sodium orthovanadate (Sigma-Aldrich, St. Louis, MO, USA), $10 \mathrm{mM}$ sodium pyrophosphate (Sigma-Aldrich, St. Louis, MO, USA). Protein concentration in the supernatant was determined using the Bradford assay (Bio-Rad Laboratories, Hercules, CA, USA). Equal amounts of total proteins $(25 \mu \mathrm{g})$ were separated on $10 \%$ SDS-PAGE and transferred onto Hybond-ECL nitrocellulose membranes (Amersham, Arlington Heights, IL, USA). The membranes were blocked with Tris-buffered salineTween 20 (TBS-T: $10 \mathrm{mM}$ Tris- $\mathrm{HCl} \mathrm{pH} \mathrm{7.6,} 150 \mathrm{mM}$ $\mathrm{NaCl}$, and $0.1 \%$ Tween 20 [USB, Cleveland, $\mathrm{OH}, \mathrm{USA}$ ]) containing 5\% nonfat dry milk (Becton Dickson and Company, Sparks, MD, USA) for $1 \mathrm{~h}$ at room temperature and incubated overnight at $4^{\circ} \mathrm{C}$ with specific primary antibodies diluted in TBS-T. The membranes were washed three times with TBS-T and then incubated with the appropriate horseradish peroxidase (HRP)-conjugated secondary antibodies for $1 \mathrm{~h}$ at room temperature. The blots were visualized using ECL detection reagents (Advansta, Menlo Park, CA, USA) and exposed to photographic films (Agfa HealthCare NV, Mortsel, Belgium). The antibody combinations and dilutions are detailed in Additional file 2: Table S2-1 and Table S2-2.

\section{Lentivirus production and titration}

Lentiviral shRNA expression vectors for non-targeting and human PKC (PRKCD) were purchased from SigmaAldrich (St. Louis, MO, USA). Lentivirus was produced by using the calcium phosphate transfection protocol and the viral titre was measured as described previously [20]. Briefly, HEK293T cells were seeded into 10-cm dishes at a density of $5 \times 10^{6}$ cells/dish and incubated overnight until they reached approximately $80 \%$ confluence. The cells 
were transfected with $10 \mu \mathrm{g}$ of the shRNA transfer vector, $7.5 \mu \mathrm{g}$ of psPAX2 viral packaging plasmid and $2.5 \mu \mathrm{g}$ of pMD2G viral envelop plasmid in a $10-\mathrm{cm}$ dish. Viral supernatants were collected at $48 \mathrm{~h}$ after transfection and used for transduction of target cells in the presence of $8 \mu \mathrm{g} / \mathrm{mL}$ polybrene (hexadimethrine bromide; SigmaAldrich, St. Louis, MO, USA) for $24 \mathrm{~h}$. Cells were cultured in the presence of $2 \mu \mathrm{g} / \mathrm{mL}$ puromycin (Sigma-Aldrich, St. Louis, MO, USA) to select shRNA-transduced cells for 3 days and then used for differentiation. The viral titre was determined by relative vector particle numbers based on virion RNA and calculated according to the following formula: relative vector particles $/ \mathrm{mL}(\mathrm{VP} / \mathrm{mL})=(\mathrm{C} \times \mathrm{D}) / \mathrm{V}$, where $\mathrm{C}=$ number of RNA copies, $\mathrm{D}=$ dilution of vector preparation (including the dilution into the PCR), and $\mathrm{V}=$ volume in $\mathrm{mL}$.

\section{Statistical analysis}

All data were expressed as the mean \pm S.E. Differences between groups were examined for statistical significance using Student's $t$-test and analysis of variance (ANOVA). The difference was considered to be significant if $P<0.05$.

\section{Results}

Up-regulation of PKC $\delta$ during osteogenic differentiation in hBMSCs

To examine the expression pattern of PKC $\delta$ during the osteogenesis of hBMSCs, we used an osteogenic differentiation model in which hBMSCs were incubated in either GM or ODM for 1, 4, 7, 10, and 14 days. Osteogenic differentiation potential of hBMSCs was confirmed by a significant increase in ALP activity and the mRNA levels of both early and late osteogenic markers, ALP and OCN, respectively (Figure $1 \mathrm{~A}$ and $\mathrm{B}$ ). This was associated with the mRNA and protein expressions of $\mathrm{PKC} \delta$ and its phosphorylation, which was significantly up-regulated at 7,10 , and 14 days after the initiation of osteogenic induction (Figure $1 \mathrm{C}$ and $\mathrm{D}$ ). Noticeably, the expression pattern of PKC $\delta$ is in stark contrast to that of PKC $\alpha$ under the same condition (Figure $1 C$ ), suggesting that among the PKC isoforms, PKC $\delta$ may act as a switch of osteogenic differentiation of hBMSCs. Taken together, these results indicate that osteogenic differentiation of hBMSCs is closely accompanied with expression and activation of PKC $\delta$.

\section{Inhibition of PKC $\delta$ activity attenuates osteogenic differentiation in hBMSCs}

To determine whether $\mathrm{PKC} \delta$ activity is required for osteogenic differentiation of hBMSCs, we treated with various concentrations of rottlerin, a $\mathrm{PKC} \delta$-specific inhibitor, during osteogenic differentiation of hBMSCs. At 7 days after induction of differentiation, the intracellular ALP activity was evaluated by colorimetric assay. Treatment with rottlerin significantly decreased ALP activity in a dose-dependent manner (Figure 2A and B). After 14 days of induction, the mineralized matrix deposition

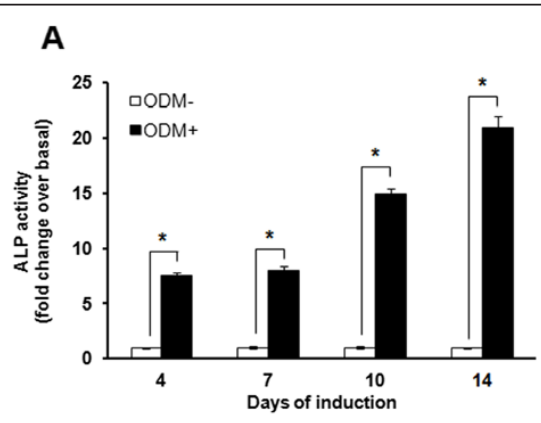

B

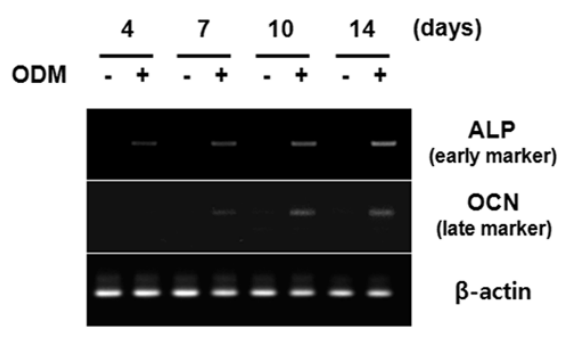

C
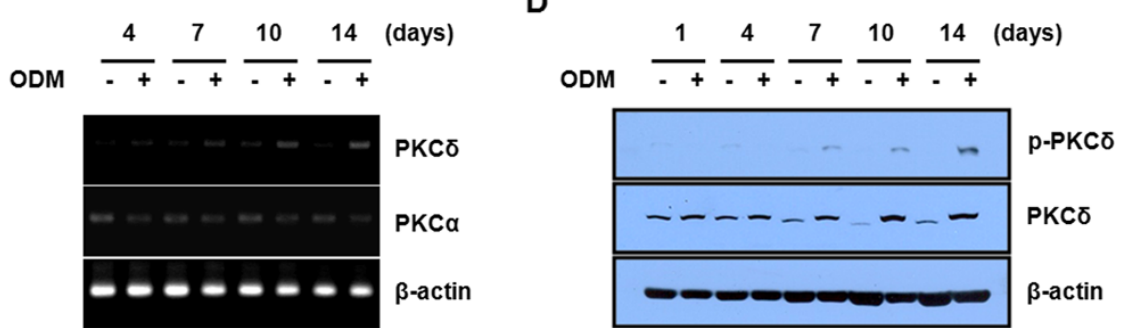

Figure 1 Up-regulation of PKC $\delta$ during osteogenic differentiation in hBMSCs. (A-D) Post-confluent hBMSCs were cultured in GM or ODM for the indicated times. The osteogenic differentiation potential of hBMSCs was estimated by ALP activity assay (A) and RT-PCR analysis of early and late osteogenic markers: ALP and OC (B) at the indicated times. (C) The mRNA expression of PKCa and $\delta$ were determined by RT-PCR at the indicated times after osteogenic induction of hBMSCs. (D) The protein level of PKC $\delta$ was determined by western blot analysis with the specified antibodies at the indicated times after osteogenic induction of hBMSCs. Data shown are means \pm S.E. ( ${ }^{*} P<0.05$ versus $\left.G M\right)$ of three independent experiments. 


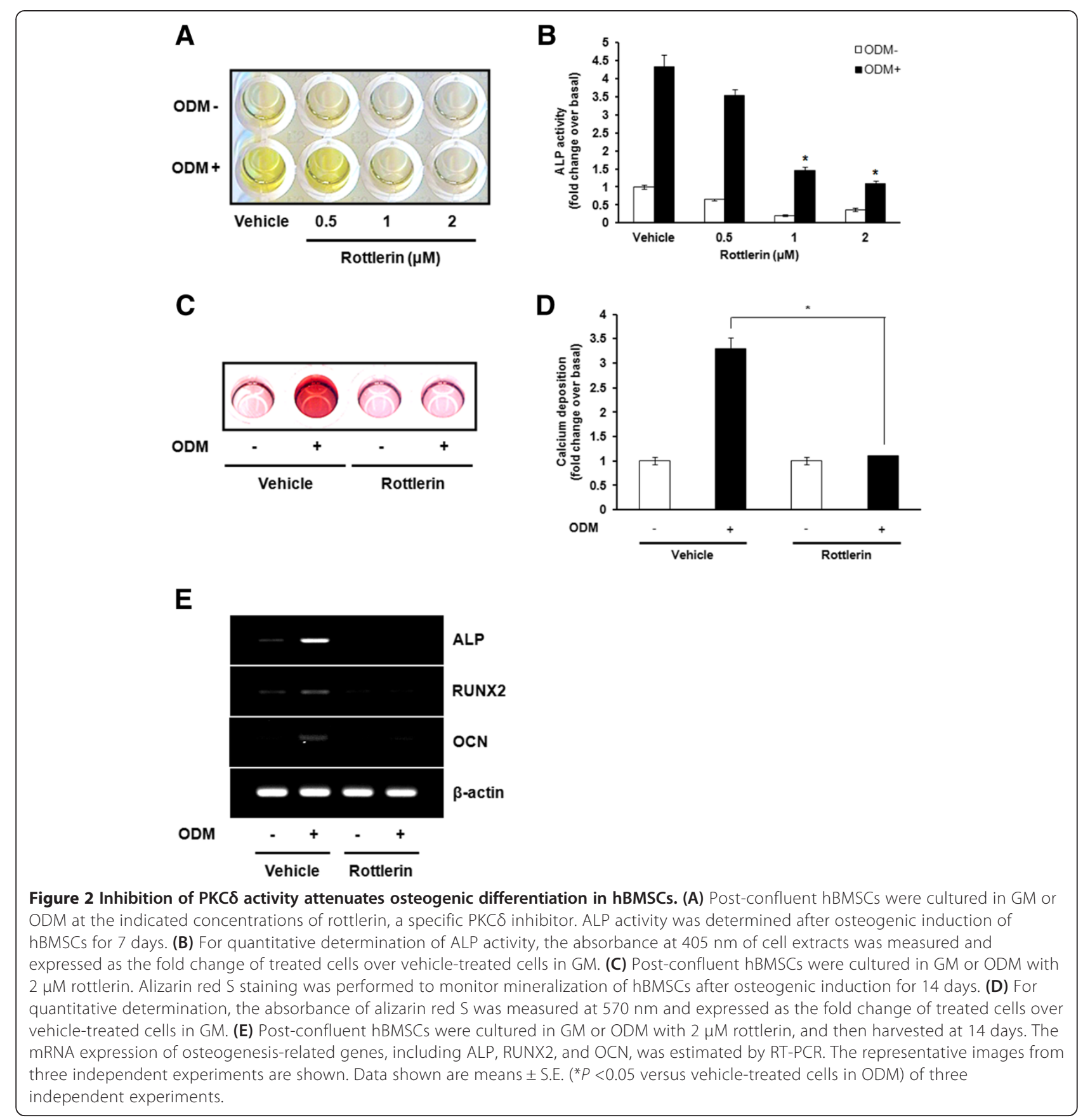

was measured by alizarin red $\mathrm{S}$ staining. Treatment with $2 \mu \mathrm{M}$ rottlerin completely blocked ECM mineralization (Figure $2 \mathrm{C}$ and $\mathrm{D}$ ). To further confirm the effects of PKC $\delta$ inhibition on osteogenic differentiation, we analyzed the gene expression pattern of major osteogenic markers such as ALP, RUNX2, and OCN. As shown in Figure 2E, the mRNA levels of ALP, RUNX2, and OCN were significantly decreased by treatment with $2 \mu \mathrm{M}$ rottlerin. These results suggest that PKC $\delta$ activation plays an important role in promoting osteogenic differentiation of hBMSCs.

\section{Knockdown of PKC $\delta$ inhibits osteogenic differentiation in hBMSCs}

Although the direct inhibition of PKC $\delta$ activity by rottlerin has been demonstrated, a major problem has been its 
limited selectivity and undesired side effects [21]. To further determine the functional role of PKC $\delta$ in osteogenic differentiation, we applied lentivirus-mediated shRNA transduction to reduce PKC $\delta$ expression in hBMSCs. The knockdown efficacy of PKC $\delta$ shRNA was confirmed by RT-PCR and western blot analysis (Figure 3A and B). After 7 days of osteogenic differentiation, the ALP activity was significantly down-regulated in PKC $\delta$ shRNA-transduced cells compared with control shRNA-transduced cells (Figure $3 \mathrm{C}$ and D). We also observed that the mineralized matrix deposition was markedly suppressed in PKC $\delta$ shRNA-transduced cells after 14 days of osteogenic induction (Figure 3E and F). Additionally, the up-regulation of osteogenesis-specific genes during osteogenic differentiation was decreased in PKC $\delta$ shRNA-transduced cells (Figure 3G). Taken together, these results support the conclusion that $\mathrm{PKC} \delta$ is necessary to trigger osteogenic differentiation of hBMSCs in a direct manner.

\section{AMPK activation is required for osteogenic differentiation of hBMSCs independently of PKC $\delta$}

AMPK has been reported as an upstream kinase of PKC $\delta$ in various cell types $[18,19]$. Based on the previous observations, we investigated whether activation of AMPK might contribute to the osteogenic differentiation of hBMSCs, together with activation of PKCס. The activation of AMPK was dramatically elevated on day 4 and maintained at a high level until day 14 after the initiation of osteogenic induction (Figure 4A). After 14 days of osteogenic induction, we observed that the mRNA levels of osteogenic differentiation markers, including ALP, RUNX2, and $\mathrm{OCN}$, were completely impaired in the cells treated with $10 \mu \mathrm{M}$ compound $\mathrm{C}$, a specific inhibitor of AMPK (Figure 4B). Subsequently, we also observed that treatment with $10 \mu \mathrm{M}$ compound C drastically reduced ALP activity (Figure $4 \mathrm{C}$ and $\mathrm{D}$ ), which is consistent with the inhibitory effect of compound $\mathrm{C}$ on the formation of mineralized ECM (Figure 4E and F). Therefore, these results suggest that AMPK is potentially capable of stimulating osteogenic differentiation of hBMSCs. However, the inhibition of AMPK activity by compound $\mathrm{C}$ did not affect the activity of PKC $\delta$ at all, as assessed by western blot analysis, indicating that there is no direct correlation between AMPK and PKC $\delta$ in osteogenesis of hBMSCs (data not shown).

\section{Suppression of PKC $\delta$-mediated osteogenic differentiation enhances the adipogenic phenotype of hBMSCs}

The clinical and experimental implications have revealed an inverse relationship between osteogenic and adipogenic differentiation in bone marrow [22-25]. The inhibition of PKC $\delta$ in mouse preadipocytes leads to enhanced adipogenic differentiation by activating the expression of adipocyte-specific genes [26,27]. Therefore, we examined whether the effect of $\mathrm{PKC} \delta$ inhibition on osteogenic differentiation causes induction of adipogenic differentiation of hBMSCs. Treatment with rottlerin induced accumulation of lipid droplets under osteogenic condition (Figure 5A). Similar to the lipid accumulation, RT-PCR results showed that the mRNA expression levels of all three adipogenic markers PPAR $\gamma, \mathrm{C} / \mathrm{EBP} \alpha$, and aP2 were increased in hBMSCs treated with rottlerin under osteogenic condition (Figure 5B). The protein expression levels of PPAR $\gamma$ and $\mathrm{C} / \mathrm{EBP} \alpha$ were also altered in the same manner as mRNA (Figure 5C). To further investigate the effect of PKC $\delta$ expression on adipogenic differentiation, we examined adipogenic differentiation potential of PKC -knockdown hBMSCs in osteogenic condition. Consistent with the effect of rottlerin that inhibition of PKC $\delta$ activity influences osteogenic differentiation by promoting PPAR $\gamma$ signaling, the lipid droplet accumulation and the expression of adipogenesis-specific markers at both the mRNA and protein levels were markedly elevated in PKC $\delta$ shRNA-transduced cells compared with control shRNA-transduced cells (Figure 5D, E, and F). Interestingly, identical to the effects of PKC $\delta$ inhibition, treatment with compound $\mathrm{C}$ increased the formation of lipid droplet and the expression of adipogenesis-specific genes at both the mRNA and protein levels under osteogenic condition (Figure 5G, H, and I). Collectively, these results strongly suggest that the effect of PKC $\delta$ inhibition on osteogenic differentiation could be sufficiently translated into adipogenic differentiation of hBMSCs.

\section{Discussion}

PKC has been implicated in the regulation of a variety of cellular processes such as cell proliferation, differentiation, apoptosis, and survival. It was previously reported that modulation of PKC activity has several therapeutic effects in cancer and other metabolic diseases such as anti-tumorigenic properties, improved glucose metabolism and cardioprotective benefits [28]. However, the exact role of PKC in bone metabolism remains to be fully elucidated. In this study, we have identified the potential role of PKC $\delta$ as a key modulator of hBMSC differentiation. Our results indicate that the expression and phosphorylation of PKC $\delta$ were markedly elevated during osteogenic differentiation of hBMSCs, leading to a significant increase in ALP activity and matrix mineralization, as well as up-regulation of the expression of osteogenesis-specific genes. Moreover, inhibition of PKC $\delta$ not only inhibited osteogenic differentiation, but also promoted lipid accumulation in hBMSCs through increased expression of adipogenesis-specific marker genes under osteogenic condition. These findings suggest that the stimulatory effect of PKC $\delta$ on osteogenic differentiation of hBMSCs appears to have occurred, at least in part by suppression of adipogenesis signaling pathway. Taken all together, this is the first experimental study to support that 
A

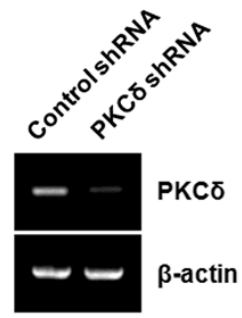

C

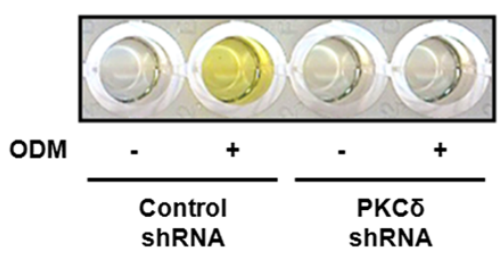

E

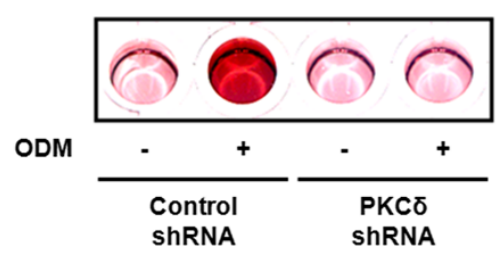

B

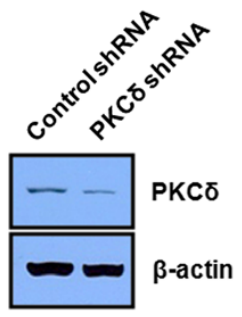

D

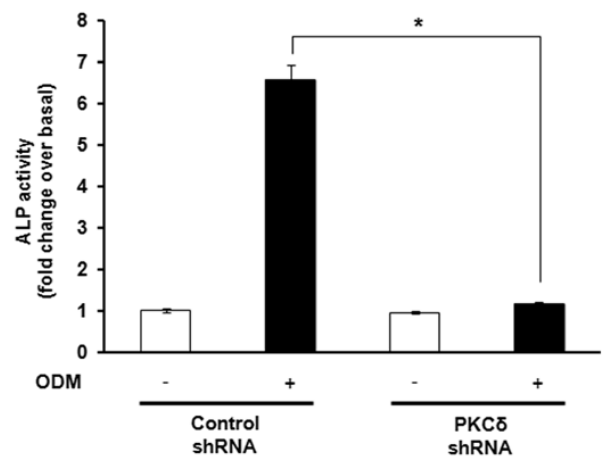

F

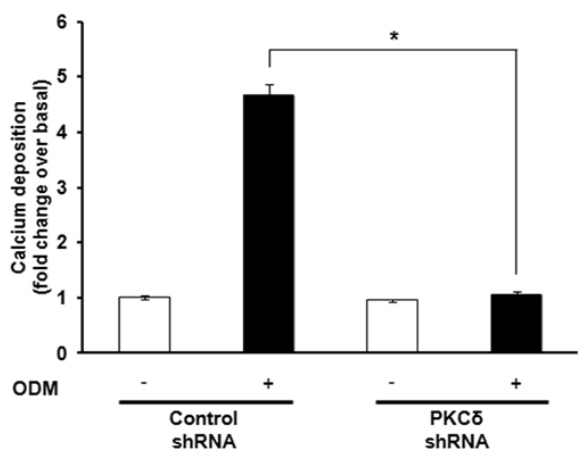

G

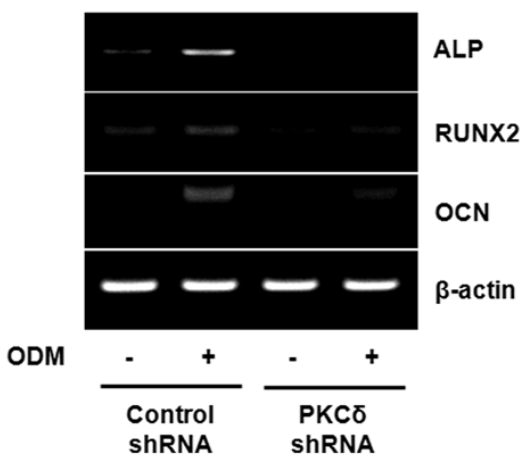

Figure $\mathbf{3}$ (See legend on next page.) 
(See figure on previous page.)

Figure 3 Knockdown of PKC $\delta$ inhibits osteogenic differentiation in hBMSCs. The knockdown efficacy of PKC $\delta$ shRNA was assessed by RT-PCR (A) and western blot analysis (B). (C) Control shRNA- or PKC $\delta$ shRNA-transduced cells were cultured in GM or ODM. ALP activity assay was determined after osteogenic induction of hBMSCs for 7 days. (D) For quantitative determination, the absorbance at $405 \mathrm{~nm}$ of ALP reaction was measured and expressed as the fold change of cultured cells over control shRNA-transduced cells in GM. (E) Control shRNA- or PKC $\delta$ shRNA-transduced cells were cultured in GM or ODM, and differentiated osteoblasts were stained with alizarin red S. (F) For quantitative determination, the absorbance of alizarin red S was measured at $570 \mathrm{~nm}$ and expressed as the fold change of cultured cells over control shRNA-transduced cells in GM. (G) The mRNA expression of ALP, RUNX2, and OCN was estimated by RT-PCR. The representative images from three independent experiments are shown. Data shown are means \pm S.E. $\left({ }^{*} P<0.05\right.$ versus control shRNA-transduced cells in ODM) of three independent experiments.

PKCס plays critical role in regulating osteogenic vs adipogenic differentiation of hBMSCs.

The expression pattern of PKC isofoms in different osteogenic precursor cell lines, including human MSCs and mouse osteoblastic MC3T3-E1 cells, has been previously investigated by several groups [29-31]. It has also been demonstrated that specific PKC isoforms have a role in regulating osteoblast activity in vitro (in cultured cells) and in vivo (in animal models). PKC $\alpha$ activation but not PKC $\beta$ suppressed osteogenic differentiation [14], whereas $\mathrm{PKC} \delta$ promoted osteogenic differentiation through the transactivation of RUNX2 [32,33]. Moreover, the decreased bone formation during embryonic skeletal development has been shown in PKC $\delta$ knockout mice, probably due to delaying the onset of Osx expression [13]. We found that the increased expression of PKC $\delta$ is in contrast to the highly restricted expression of PKC $\alpha$ during osteogenic induction of hBMSCs (Figure 1). This is consistent with the previous findings that overexpression of $\mathrm{PKC} \delta$ significantly decreased $\mathrm{PKC} \alpha$ activity, while expression of dominant negative mutant of PKC significantly increased it in vitro [34]. The expression pattern of $\mathrm{PKC} \delta$ positively correlates with the rapid induction of ALP acitivty and the upregulation of early and late osteogenic marker genes, ALP and OCN, respectively. Therefore, these data suggest a potential role of PKC $\delta$ in the regulatory mechanism of osteogenic differentiation.

In the present study, suppression of PKC $\delta$ activity with a specific inhibitor, rottlerin, or depletion of PKC $\delta$ by lentiviral shRNA in hBMSCs inhibited induction of osteogenic differentiation (Figure 2 and Figure 3). We found that activation of PKC $\delta$ during osteogenic differentiation of hBMSCs leads to increased expression of RUNX2 and its downstream targets, ALP and OCN, which are known to be regulated by RUNX2. Moreover, inhibition of PKC $\delta$ using rottlerin or PKC $\delta$ shRNA completely reversed the osteogenic response of hBMSCs, suggesting that $\mathrm{PKC} \delta$ functions as a potent activator of RUNX2 expression in bone development. Considerable evidence now suggests that $\mathrm{PKC} \delta$-dependent mechanism plays an important role in bone development. Several studies have indicated that osteogenic differentiation is associated with an increase in RUNX2 transcriptional activity through phosphorylation of RUNX2 at key residues by $P K C \delta$, without changing the protein levels of RUNX2 [35,36]. The translocation of PKC $\delta$ from the cytoplasm to the nucleus in response to osteogenic condition could indeed influence the phosphorylation status and modulation of RUNX2 DNA-binding activity, which are concomitant with the enhanced $\mathrm{OCN}$ gene transcription $[32,33,37]$. Thus, although the functional interaction between PKC $\delta$ and RUNX2 was not assessed in the present study, it is possible that PKC $\delta$ could be directly responsible for enhancing osteogenic differentiation through both the regulation of RUNX2 expression and transcriptional activity in hBMSCs.

In terms of identifying the novel regulatory mechanisms that mediate the stimulatory effect of PKC $\delta$ on osteogenic differentiation of hBMSCs, it is worth noting that the effect of PKC $\delta$ on the osteogenic differentiation of hBMSCs occurs in parallel with an increased AMPK activity (Figure 4). Indeed, we found that AMPK activation increased markedly during osteogenic differentiation of hBMSCs and inhibition of AMPK reduced the gene expression of osteogenic markers in osteogenesis assays in vitro, including ALP activity and matrix mineralization. These results indicate an important role for AMPK in osteogenic differentiation of hBMSCs. A positive role of AMPK in driving and sustaining osteogenic differentiation is supported by the prior reports that AMPK activation facilitates bone formation by upregulating expression of osteogenic lineage-specific genes [38,39]. Since both PKC $\delta$ and AMPK have a stimulating effect on osteogenic differentiation of hBMSCs, whether a direct connection between PKC $\delta$ and AMPK may synergize to accelerate osteogenic program of hBMSCs is a remaining question. Interestingly, AMPK was found to be associated with PKC $\delta$ activation in monocytic and lymphocytic cells [19]. In this study, although PKC $\delta$ and AMPK activation have identical effects on the regulation of hBMSC differentiation, no direct correlation was found between these two kinases. Consequently, $\mathrm{PKC} \delta$ appears to coordinate osteogenic differentiation in hBMSCs independently of AMPK pathway. Further study is required to elucidate the novel upstream and 


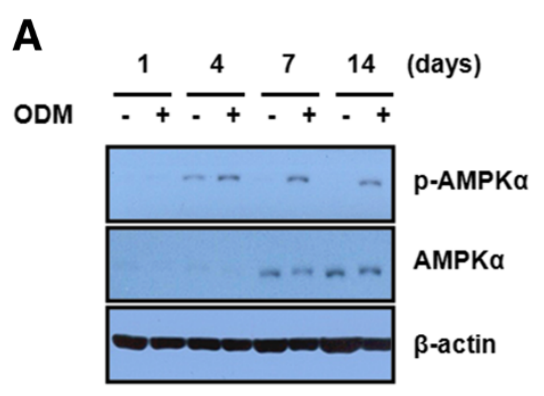

B
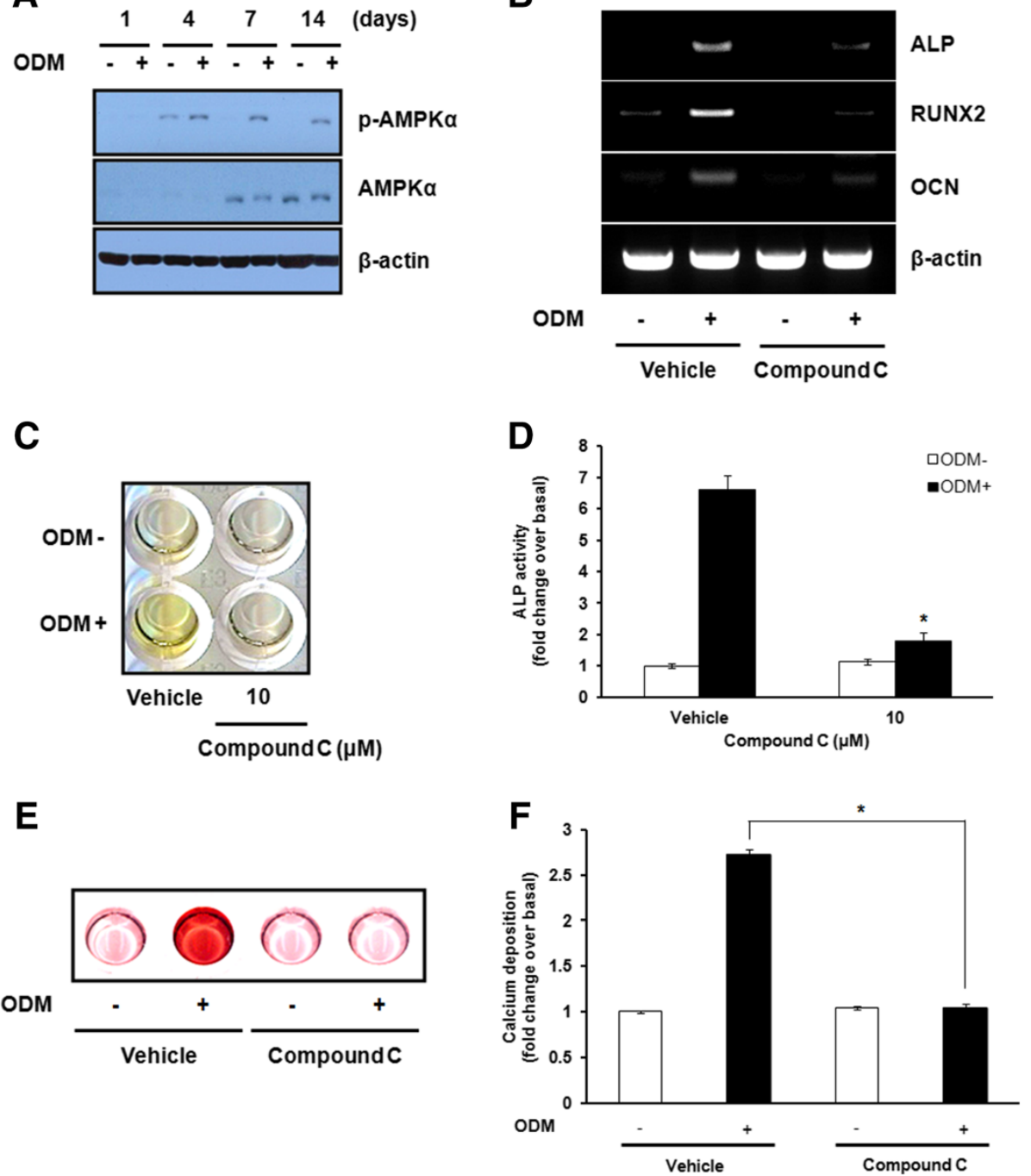

Figure 4 AMPK activation is required for osteogenic differentiation in hBMSCs independently of PKC $\delta$. (A) Post-confluent hBMSCs were cultured in GM or ODM for the indicated times. Cell lysates were prepared and subjected to western blot analysis using the indicated antibodies. (B) Post-confluent hBMSCs were cultured in GM or ODM with $10 \mu \mathrm{M}$ compound C, a specific inhibitor of AMPK, and then harvested after 14 days. The mRNA expression of ALP, RUNX2, and OCN was estimated by RT-PCR. (C) Post-confluent hBMSCs were cultured in GM or ODM with $10 \mu M$ compound C. After 7 days of osteogenic induction, ALP activity was determined by the ALP activity assay. (D) For quantitative determination, the absorbance at $405 \mathrm{~nm}$ of ALP reaction was measured and expressed as the fold change of treated cells over vehicle-treated cells in GM. (E) Post-confluent hBMSCs were cultured in GM or ODM with $10 \mu \mathrm{M}$ compound C. Differentiated osteoblasts were stained with alizarin red S after 14 days of osteogenic induction. (F) For quantitative determination, the absorbance of alizarin red $\mathbf{S}$ was measured at $570 \mathrm{~nm}$ and expressed as the fold change of treated cells over vehicle-treated cells in GM. The representative images from three independent experiments are shown. Data shown are means \pm S.E. ( ${ }^{*} P<0.05$ versus vehicle-treated cells in ODM) of three independent experiments.

downstream effectors of $\mathrm{PKC} \delta$ during osteogenic differentiation of hBMSCs.

It is also noteworthy that inhibition of PKC $\delta$ using rottlerin or PKC $\delta$ shRNA suppressed osteogenesis but promoted adipogenesis of hBMSCs (Figure 5). These biphasic effects of PKC $\delta$ on hBMSC differentiation are likely to be partially explained by the inverse relationship between osteogenesis and adipogenesis in the bone marrow. It has been well established that the balance between osteogenesis and adipogenesis in MSCs depends on different signaling pathways that converge on the regulation of the two master transcription factors RUNX2 and PPARy. The osteogenic and adipogenic signaling pathway may contribute to RUNX2 and PPAR $\gamma$ expression through a mutually negative interconnection [22-25]. Dysregulation of bone and fat formation is 


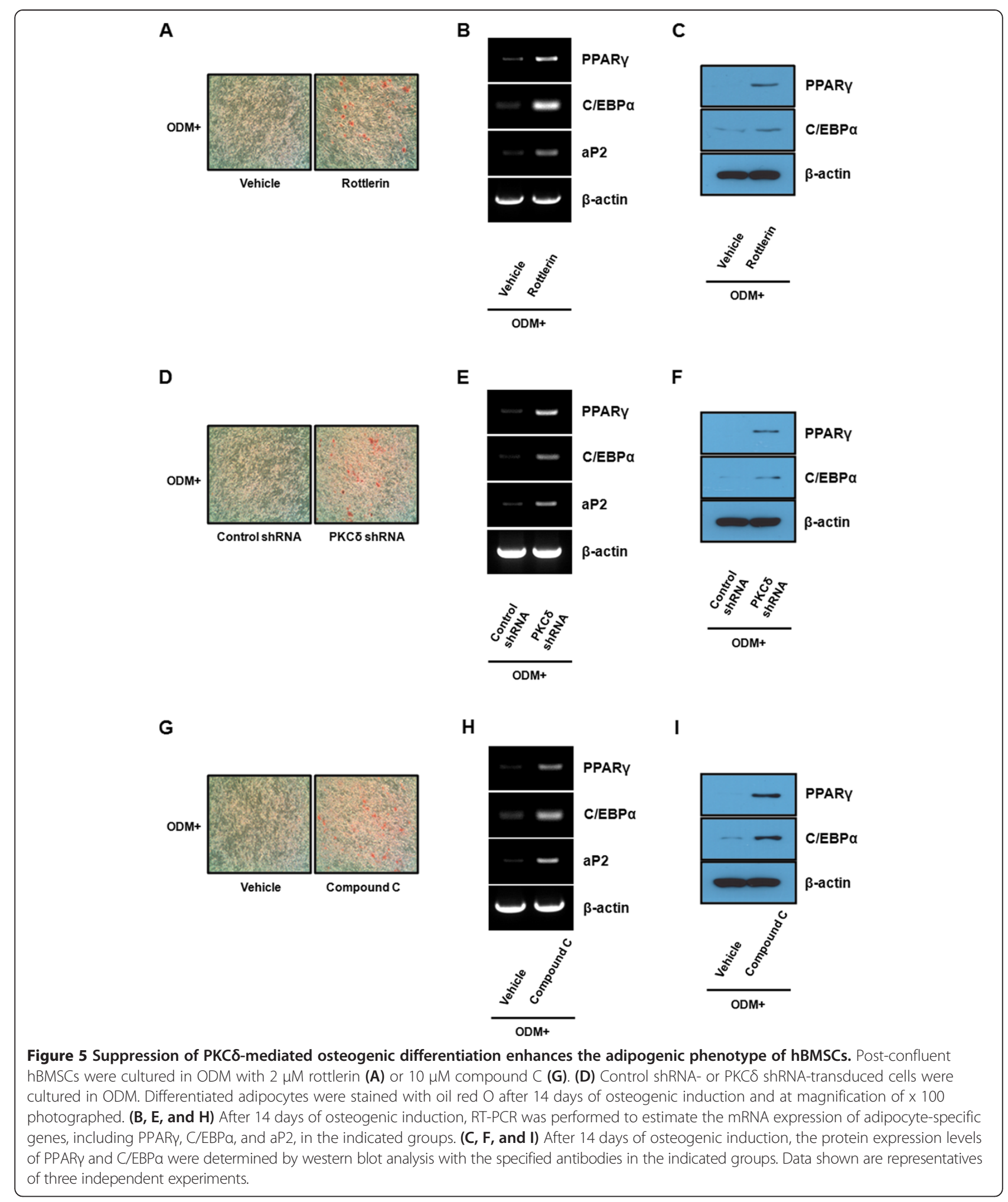

implicated with a high incidence of both osteoporosis and obesity $[40,41]$. We found PKC $\delta$ inhibition during osteogenic differentiation of hBMSCs resulted in a pronounced decrease in the expression of osteogenic transcription factor RUNX2 and up-regulation of major adipogenic transcription factors PPAR $\gamma$ and C/EBP $\alpha$. These effects lead to suppression of bone formation and decrease of bone mineral content, and the formation of 


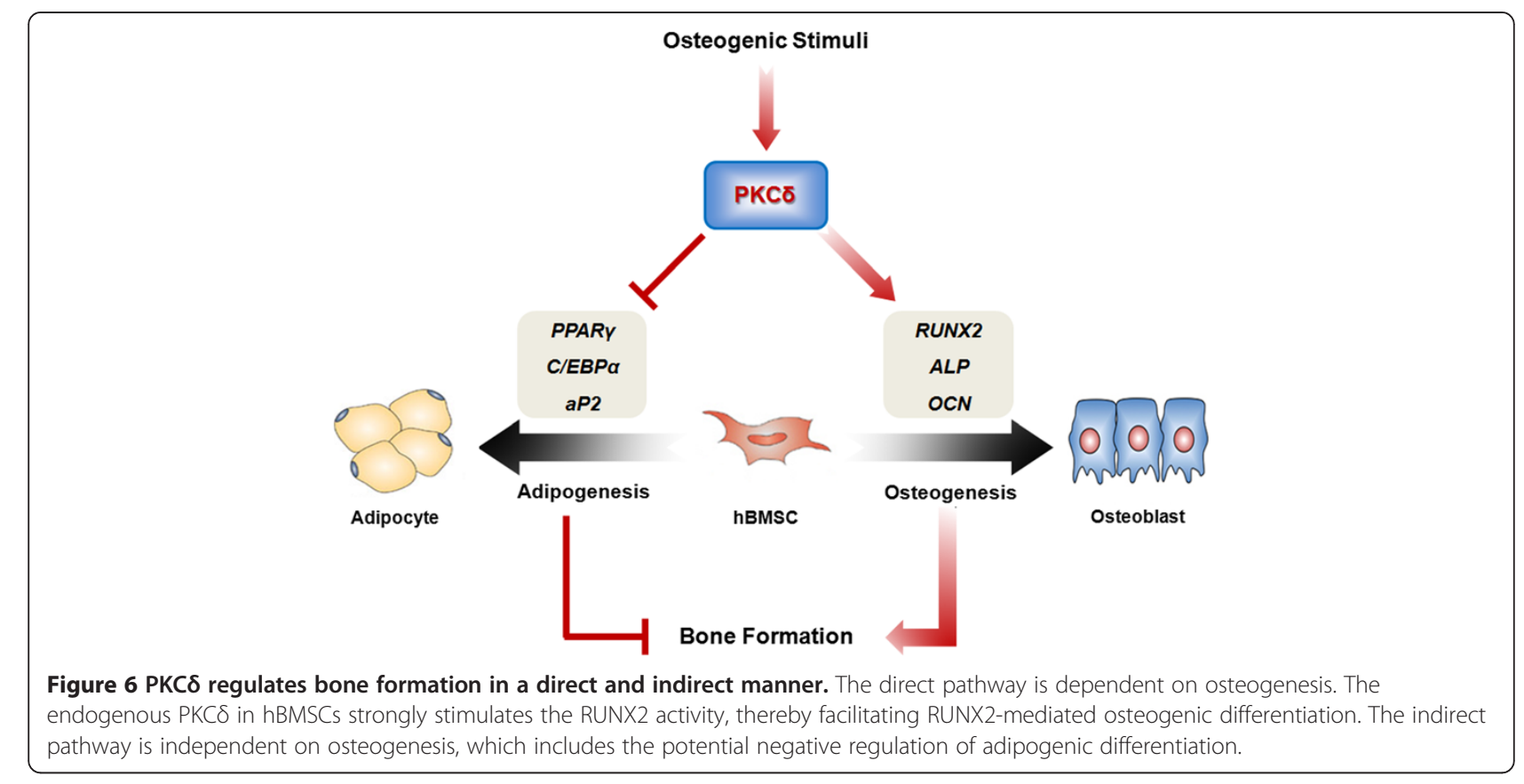

adipocyte-like phenotype containing intracellular lipid droplets in hBMSCs. Thus, it is plausible that PKC $\delta$ increases endogenous RUNX2 transcriptional activity, and up-regulated RUNX2 facilitates RUNX2-dependent gene transcription and osteogenesis, thereby repressing PPAR $\gamma$ dependent gene transcription and adipogenesis (Figure 6). Meanwhile, it has been reported that the lineage commitment and differentiation of transformed haematopoietic progenitors is determined by the level of PKC activity, suggesting that the regulation of PKC activity is critical for governing the differentiation capacity of haematopoietic progenitor cells [42]. Taken together, these findings emphasize the important physiological effect of PKC $\delta$ on the relationship between bone and fat metabolism. Interestingly, identical to the effects of PKC $\delta$ inhibition, the adipogenic phenotype as a result of AMPK inhibition during in vitro osteogenesis of hBMSCs has also been observed (Figure 5). Our data are in agreement with the involvement of AMPK in stem cell differentiation [43], which supports evidence that AMPK may inhibit adipogenic differentiation by shifting stem cell fate toward osteogenic differentiation via a $\mathrm{PKC} \delta$-independent pathway.

\section{Conclusions}

The results of present study demonstrate that PKC $\delta$ and AMPK has a crucial role in regulating the balance between osteogenesis and adipogenesis of hBMSCs. Since appropriate management of hBMSC differentiation is important for the development and maintenance of healthy bones, this study might provide a new insight into the regulatory mechanisms of hBMSC differentiation, further encouraging novel therapeutic strategies for improving bone regeneration.

\section{Additional files}

Additional file 1: Table S1. List of all the primer sequences used for RT-PCR analysis.

Additional file 2: Table S2-1. List of primary antibodies used for western blot analysis. Table S2-2. List of secondary antibodies used for western blot analysis.

\section{Abbreviations}

MSCs: Mesenchymal stem cells; PKC: Protein kinase C; hBMSCs: Human bone marrow-derived mesenchymal stem cells; hMSCs: Human mesenchymal stem cells; ALP: Alkaline phosphatase; OCN: Osteocalcin; RUNX2: Runt-related transcription factor 2; OSX: Osterix; DAG: Diacylglycerol; GM: Growth medium; a-MEM: a-Minimum essential medium; FBS: Fetal bovine serum; DMEM: Dulbecco's modified Eagle's medium; ODM: Osteogenic differentiation medium; ECM: Extracellular matrix; PPARY: Peroxisome proliferator-activated receptor $\gamma$; C/EBPa: CCAAT/enhancer-binding protein $a$; aP2: Adipocyte fatty acid binding protein; TBS-T: Tris-buffered saline-Tween 20; HRP: Horseradish peroxidase; ANOVA: Analysis of variance.

\section{Competing interests}

The authors declare that they have no competing interests.

\section{Authors' contributions}

SL and DK conception and design of research; SL, H-YC, and HTTB performed experiments; SL and DK analyzed data; SL and DK interpreted results of experiments; SL and DK prepared figures; SL and DK drafted manuscript; SL and DK edited and revised manuscript; SL and DK approved final version of manuscript. All authors read and approved the final manuscript.

\section{Acknowledgements}

This work was supported by Basic Science Research Program through the National Research Foundation of Korea (NRF) funded by the Ministry of Education, Science and Technology (No. 2011-0025290). 
Received: 10 July 2014 Accepted: 5 November 2014 Published online: 25 November 2014

\section{References}

1. Chamberlain G, Fox J, Ashton B, Middleton J: Concise review: mesenchymal stem cells: their phenotype, differentiation capacity, immunological features, and potential for homing. Stem Cells 2007, 25(11):2739-2749.

2. Pittenger MF, Mackay AM, Beck SC, Jaiswal RK, Douglas R, Mosca JD, Moorman MA, Simonetti DW, Craig S, Marshak DR: Multilineage potential of adult human mesenchymal stem cells. Science 1999, 284(5411):143-147.

3. Uccelli A, Moretta L, Pistoia V: Mesenchymal stem cells in health and disease. Nat Rev Immunol 2008, 8(9):726-736.

4. Giordano A, Galderisi U, Marino IR: From the laboratory bench to the patient's bedside: an update on clinical trials with mesenchymal stem cells. J Cell Physiol 2007, 211(1):27-35.

5. Long F: Building strong bones: molecular regulation of the osteoblast lineage. Nat Rev Mol Cell Biol 2012, 13(1):27-38.

6. Stein GS, Lian JB, Van Wijnen AJ, Stein JL, Montecino M, Javed A, Zaidi SK, Young DW, Choi JY, Pockwinse SM: Runx2 control of organization, assembly and activity of the regulatory machinery for skeletal gene expression. Oncogene 2004, 23(24):4315-4329.

7. Arvidson K, Abdallah BM, Applegate LA, Baldini N, Cenni E, Gomez-Barrena E, Granchi D, Kassem M, Konttinen YT, Mustafa K, Pioletti DP, Sillat T, Finne-Wistrand A: Bone regeneration and stem cells. J Cell Mol Med 2011, 15(4):718-746.

8. Papachroni KK, Karatzas DN, Papavassiliou KA, Basdra EK, Papavassiliou AG Mechanotransduction in osteoblast regulation and bone disease. Trends Mol Med 2009, 15(5):208-216.

9. Griner EM, Kazanietz MG: Protein kinase $\mathrm{C}$ and other diacylglycerol effectors in cancer. Nat Rev Cancer 2007, 7(4):281-294.

10. Teicher BA: Protein kinase $\mathrm{C}$ as a therapeutic target. Clin Cancer Res 2006, 12(18):5336-5345.

11. Geraldes $P$, King GL: Activation of protein kinase $C$ isoforms and its impact on diabetic complications. Circ Res 2010, 106(8):1319-1331.

12. Rosse C, Linch M, Kermorgant S, Cameron AJ, Boeckeler K, Parker PJ: PKC and the control of localized signal dynamics. Nat Rev Mol Cell Biol 2010, 11(2):103-112.

13. Tu X, Joeng KS, Nakayama KI, Nakayama K, Rajagopal J, Carroll TJ, McMahon AP, Long F: Noncanonical Wnt signaling through G protein-linked PKCdelta activation promotes bone formation. Dev Cell 2007, 12(1):113-127.

14. Nakura A, Higuchi C, Yoshida K, Yoshikawa H: PKCalpha suppresses osteoblastic differentiation. Bone 2011, 48(3):476-484.

15. Park KH, Han DI, Rhee YH, Jeong SJ, Kim SH, Park YG: Protein kinase C betall and delta/theta play critical roles in bone morphogenic protein-4stimulated osteoblastic differentiation of MC3T3-E1 cells. Biochem Biophys Res Commun 2010, 403(1):7-12

16. Khor EC, Abel T, Tickner J, Chim SM, Wang C, Cheng T, Ng B, Ng PY, Teguh DA, Kenny J, Yang X, Chen H, Nakayama KI, Nakayama K, Pavlos N, Zheng $\mathrm{MH}, \mathrm{Xu}$ J: Loss of protein kinase C-delta protects against LPS-induced osteolysis owing to an intrinsic defect in osteoclastic bone resorption. PLoS One 2013, 8(8):e70815.

17. Jeyabalan J, Shah M, Viollet B, Chenu C: AMP-activated protein kinase pathway and bone metabolism. J Endocrinol 2012, 212(3):277-290.

18. Bair AM, Thippegowda PB, Freichel M, Cheng N, Ye RD, Vogel SM, Yu Y, Flockerzi V, Malik AB, Tiruppathi C: Ca2+ entry via TRPC channels is necessary for thrombin-induced NF-kappaB activation in endothelial cells through AMP-activated protein kinase and protein kinase Cdelta. J Biol Chem 2009, 284(1):563-574.

19. Mehla R, Bivalkar-Mehla S, Zhang R, Handy I, Albrecht H, Giri S, Nagarkatti $P$, Nagarkatti M, Chauhan A: Bryostatin modulates latent HIV-1 infection via PKC and AMPK signaling but inhibits acute infection in a receptor independent manner. PLoS One 2010, 5(6):e11160

20. Kutner RH, Zhang XY, Reiser J: Production, concentration and titration of pseudotyped HIV-1-based lentiviral vectors. Nat Protoc 2009, 4(4):495-505.

21. Soltoff SP: Rottlerin: an inappropriate and ineffective inhibitor of PKCdelta. Trends Pharmacol Sci 2007, 28(9):453-458.

22. Takada I, Kouzmenko AP, Kato S: Wnt and PPARgamma signaling in osteoblastogenesis and adipogenesis. Nat Rev Rheumatol 2009, 5(8):442-447.
23. Duque G: Bone and fat connection in aging bone. Curr Opin Rheumatol 2008, 20(4):429-434.

24. Harada S, Rodan GA: Control of osteoblast function and regulation of bone mass. Nature 2003, 423(6937):349-355.

25. Rosen CJ, Klibanski A: Bone, fat, and body composition: evolving concepts in the pathogenesis of osteoporosis. Am J Med 2009, 122(5):409-414.

26. Zhou Y, Wang D, Li F, Shi J, Song J: Different roles of protein kinase C-betal and -delta in the regulation of adipocyte differentiation. Int J Biochem Cell Biol 2006, 38(12):2151-2163.

27. Schmitz-Peiffer $\mathrm{C}$ : The tail wagging the dog-regulation of lipid metabolism by protein kinase C. FEBS J 2013, 280(21):5371-5383.

28. Mochly-Rosen D, Das K, Grimes KV: Protein kinase C, an elusive therapeutic target? Nat Rev Drug Discov 2012, 11(12):937-957.

29. Liu J, Someren E, Mentink A, Licht R, Dechering K, van Blitterswijk C, de Boer $J$ : The effect of PKC activation and inhibition on osteogenic differentiation of human mesenchymal stem cells. J Tissue Eng Regen Med 2010, 4(5):329-339.

30. Lampasso JD, Chen W, Marzec N: The expression profile of PKC isoforms during MC3T3-E1 differentiation. Int J Mol Med 2006, 17(6):1125-1131.

31. Sanders JL, Stern PH: Expression and phorbol ester-induced downregulation of protein kinase C isozymes in osteoblasts. J Bone Miner Res 1996, 11(12):1862-1872.

32. Kim HJ, Kim JH, Bae SC, Choi JY, Ryoo HM: The protein kinase C pathway plays a central role in the fibroblast growth factor-stimulated expression and transactivation activity of Runx2. J Biol Chem 2003, 278(1):319-326.

33. Kim BG, Kim HJ, Park HJ, Kim YJ, Yoon WJ, Lee SJ, Ryoo HM, Cho JY: Runx2 phosphorylation induced by fibroblast growth factor-2/protein kinase $C$ pathways. Proteomics 2006, 6(4):1166-1174.

34. Murakami M, Horowitz A, Tang S, Ware JA, Simons M: Protein kinase C (PKC) delta regulates PKCalpha activity in a Syndecan-4-dependent manner. J Biol Chem 2002, 277(23):20367-20371.

35. Shui C, Spelsberg TC, Riggs BL, Khosla S: Changes in Runx2/Cbfa1 expression and activity during osteoblastic differentiation of human bone marrow stromal cells. J Bone Miner Res 2003, 18(2):213-221.

36. Jonason $J H$, Xiao G, Zhang M, Xing L, Chen D: Post-translational regulation of Runx2 in Bone and Cartilage. J Dent Res 2009, 88(8):693-703.

37. Lima F, Niger C, Hebert C, Stains JP: Connexin43 potentiates osteoblast responsiveness to fibroblast growth factor 2 via a protein kinase C-delta/ Runx2-dependent mechanism. Mol Biol Cell 2009, 20(11):2697-2708.

38. Pantovic A, Krstic A, Janjetovic K, Kocic J, Harhaji-Trajkovic L, Bugarski D, Trajkovic V: Coordinated time-dependent modulation of AMPK/Akt/mTOR signaling and autophagy controls osteogenic differentiation of human mesenchymal stem cells. Bone 2013, 52(1):524-531.

39. Kim EK, Lim S, Park JM, Seo JK, Kim JH, Kim KT, Ryu SH, Suh PG: Human mesenchymal stem cell differentiation to the osteogenic or adipogenic lineage is regulated by AMP-activated protein kinase. J Cell Physiol 2012, 227(4):1680-1687.

40. Rosen CJ, Bouxsein ML: Mechanisms of disease: is osteoporosis the obesity of bone? Nat Clin Pract Rheumatol 2006, 2(1):35-43.

41. Zhao LJ, Jiang H, Papasian CJ, Maulik D, Drees B, Hamilton J, Deng HW: Correlation of obesity and osteoporosis: effect of fat mass on the determination of osteoporosis. J Bone Miner Res 2008, 23(1):17-29.

42. Rossi F, McNagny M, Smith G, Frampton J, Graf T: Lineage commitment of transformed haematopoietic progenitors is determined by the level of PKC activity. EMBO J 1996, 15(8):1894-1901.

43. Chen H, Liu X, Cao J, Zhang L, Hu X, Wang J: Role of SIRT1 and AMPK in mesenchymal stem cells differentiation. Ageing Res Rev 2014, 13:55-64.

doi:10.1186/s12860-014-0042-4

Cite this article as: Lee et al:: The osteogenic or adipogenic lineage commitment of human mesenchymal stem cells is determined by protein kinase C delta. BMC Cell Biology 2014 15:42. 